\title{
O impacto do diabetes mellitus na qualidade de vida de pacientes da Unidade Básica de Saúde
}

\author{
Luciana Sabadini Bernini, Silvia Regina Barrile, Aline Faulin Mangili, Eduardo Aguilar Arca, \\ Rinaldo Correr, Maria Amélia Ximenes, Débora Neves, Camila Gimenes
}

Universidade Sagrado Coração - USC, Bauru, São Paulo, Brasil.

\begin{abstract}
Resumo: Introdução: O diabetes mellitus tipo 2 (DM2) é um distúrbio crônico com morbimortalidade que limita a qualidade de vida devido às complicações. Objetivo: Avaliar, por meio de questionários, os aspectos físicos, emocionais e sociais que envolvem a doença e a qualidade de vida de pacientes com DM tipo 2 de uma unidade básica de saúde (UBS) de Bauru (SP). Método: Os participantes (diabéticos tipo 2, acima de 40 anos, ambos os sexos, adscritos na UBS Vila Cardia, em Bauru [SP]) responderam aos questionários B-PAID (perspectiva do impacto do DM na qualidade de vida e aspectos emocionais), DQOL (satisfação, impacto, preocupação social/vocacional relacionada ao DM) e QAD (autocuidado). Os dados foram apresentados de forma descritiva, tendo sido correlacionado o tempo de DM com escores dos questionários B-PAID e DQOL pela correlação de Spearman $(\mathrm{p}<0,05)$. Resultados: A amostra foi de 59 indivíduos, com idade de $63,5 \pm 10$ anos, peso de $79 \pm 13 \mathrm{~kg}$ e índice de massa corporal de $29 \pm 4 \mathrm{~kg} / \mathrm{m}^{2}$. O questionário B-PAID indicou escore de 19 (11 a 48) pontos, demonstrando baixo sofrimento emocional, o DQOL indicou que as preocupações mais frequentes foram complicações decorrentes do DM e o QAD encontrou menor aderência no item "Ingerir doces" e maior no "Tomar o número indicado de comprimidos". Houve correlação positiva do tempo de DM com o escore do B-PAID, isto é, quanto menor o tempo de doença, menor o sofrimento emocional. Conclusão: Espera-se que este estudo contribua para trazer informações sobre os pacientes e direcionar intervenções de saúde, visando melhorar a qualidade de vida e o autocuidado do DM2.
\end{abstract}

Palavras-chave: Diabetes Mellitus, Avaliação em Saúde, Qualidade de Vida.

\section{The impact of diabetes mellitus on the quality of life of patients of Primary Health Care}

\begin{abstract}
Introduction: Diabetes Mellitus type 2 (DM2) is a chronic disorder with morbidity and mortality, which limits the quality of life due to complications. Objective: To evaluate through questionnaires the physical, emotional and social issues surrounding the disease and quality of life of patients with type 2 DM of a Basic Health Unit (BHU) of Bauru/SP. Method: The participants (patients type 2 DM, over 40 years, both genders, registered in the BHU Vila Cardia, Bauru-SP) answered the B-PAID questionnaire (DM impact perspective on quality of life and emotional aspects); DQOL (satisfaction, impact, social/ vocational preoccupation related to DM) and QAD (self-care). The data were presented descriptively, it was correlated with the time of DM with scores of B-PAID and DQOL questionnaires by Spearman correlation $(\mathrm{p}<0.05)$. Results: The sample consisted of 59 individuals, aged $63.5 \pm 10$ years, weight $79 \pm 13 \mathrm{~kg}$, BMI $29 \pm 4 \mathrm{~kg} / \mathrm{m}^{2}$. The questionnaire B-PAID indicated score of 19 (11-48) points evidencing low emotional suffering, the DQOL indicated that the most frequent preoccupation were the complications of DM and the QAD found lower adherence in "sweet ingest" and higher in "ingesting the indicated number of drugs". There was a positive correlation of DM time with the score of B-PAID, the shorter the duration of disease, less emotional suffering. Conclusion: It is expected that this study will contribute to provide information about the patients and direct health interventions, aiming to improve the quality of life and self-care of type 2 DM.
\end{abstract}

Keywords: Diabetes Mellitus, Health Evaluation, Quality of Life.

Autor para correspondência: Camila Gimenes, Universidade Sagrado Coração, Rua Irmã Arminda, 10-50, Jardim Brasil, CEP 17011-160, Bauru, SP, Brasil, e-mail: professoracamilagimenes@gmail.com

Recebido em Mar. 24, 2016; 1ª Revisão em Dez. 5, 2016; Aceito em Mar. 15, 2017. 


\section{Introdução}

O aumento da expectativa de vida do brasileiro e o envelhecimento populacional, nestas últimas décadas, acarretaram mudanças epidemiológicas no Brasil, como a redução da morbidade e mortalidade por doenças infecciosas e o aumento da prevalência de doenças crônicas não transmissíveis, como o diabetes mellitus (DM). Isso traz novas e crescentes necessidades de atenção à saúde e onera os serviços de saúde pública (DUNCAN et al., 2012).

Segundo a International Diabetes Federation (IDF), existem aproximadamente 387 milhóes de pessoas com DM no mundo, e esse número vem aumentando significativamente, principalmente o DM tipo 2 (DM2), podendo, em 2035, chegar a 592 milhóes de pessoas. Em 2014, o custo com despesas de saúde em indivíduos com DM foi de aproximadamente 612 milhôes de dólares (INTERNATIONAL..., 2014). A Organização Mundial da Saúde apontou o Brasil como o oitavo país com mais prevalência da doença (BRASIL, 2015; ORGANIZAÇÃO..., 2010).

O DM é um distúrbio metabólico, crônico, caracterizado pelo aumento no nível de glicose no sangue (hiperglicemia) e que limita a qualidade de vida dos pacientes (ASSOCIAÇÃO..., 2015; ARSA et al., 2009). O DM2 é o mais comum, acomete indivíduos acima de 40 anos e pode estar associado a fatores de risco modificáveis, como sobrepeso ou obesidade, dislipidemia, disfunção endotelial, hipertensão arterial sistêmica (HAS), e a fatores não modificáveis, como antecedentes familiares e idade (ARSA et al., 2009; ZABAGLIA et al., 2009; SOCIEDADE..., 2014a). Todos esses fatores de risco estão associados ao estilo de vida, como dieta rica em gorduras saturadas, colesterol, tabagismo, consumo de álcool em excesso e sedentarismo. Sintomas frequentes do DM são aumento de peso, dores nos membros inferiores, manifestaçôes musculoesqueléticas, alterações visuais, sede e diurese excessiva, os quais aparecem de maneira lenta, o que favorece o surgimento de complicações com o passar do tempo (SILVA; SKARE, 2012; ARSA et al., 2009; ZABAGLIA et al., 2009; SOCIEDADE..., 2014b). A equipe de saúde, em especial na Atenção Básica, deve prestar sua contribuição no controle desses fatores e sintomas apresentados que implicam modificação de hábitos, rotinas e papéis ocupacionais (CORDEIRO, 2007; DUARTE; UCHÔA-FIGUEIREDO, 2010).

O controle do DM pode proporcionar melhora na qualidade de vida dos pacientes e, para que isso ocorra, deve-se investir em trabalhos de promoção de saúde e intervençóes precoces. Estudos sugerem a atuação transdisciplinar com tais pacientes a fim de buscar soluçóes coletivas e abrir caminho para um exercício democrático na tomada de decisóes, tanto na prevenção quanto no tratamento (TANAKA; MELO, 2000; DUARTE; UCHÔA-FIGUEIREDO, 2010).

Existem instrumentos específicos para indivíduos com DM que avaliam o impacto causado pela doença no cotidiano e na qualidade de vida e aspectos como o bem-estar físico, fatores biológicos, psicológicos e sociais, domínios importantes que contemplam os estudos de qualidade de vida (CORRER et al., 2013).

Neste estudo foram utilizados questionários validados e confiáveis com a finalidade de proporcionar autoconhecimento e contribuir para intervençôes interdisciplinares adequadas. O objetivo da pesquisa foi avaliar, por meio de questionários, os aspectos físicos, emocionais e sociais que envolvem a doença e a qualidade de vida de pacientes com DM2 de uma Unidade Básica de Saúde (UBS) da cidade de Bauru (SP).

\section{Método}

Trata-se de um estudo observacional transversal. A amostra foi constituída por pacientes com DM2, acima de 40 anos, de ambos os sexos, adscritos na Unidade Básica de Saúde da Vila Cardia, na cidade de Bauru (SP). Foram excluídos indivíduos com tempo de diagnóstico de DM inferior a um ano e aqueles que não concordaram em responder aos questionários.

O projeto foi aprovado pelo Comitê de Ética e Pesquisa em Seres Humanos da Universidade Sagrado Coração, sob o parecer n $n^{\circ}$ 1.079.203.

Foi utilizada uma ficha de avaliaçáo sistematizada com dados sociodemográficos, estilo de vida como hábitos de fumar, ingerir bebida alcoólica e prática de atividades físicas. Foram coletados dados antropométricos, como peso, altura, índice de massa corporal (IMC), circunferência abdominal (CA) e, além disso, história clínica, como antecedentes familiares, doenças associadas e tempo de diagnóstico de DM. Em seguida, foram aplicados três questionários específicos para indivíduos com DM, validados e traduzidos para o português.

O B-PAID (Problems Areas in Diabetes) é um questionário adaptado para autoavaliação, composto de 20 questóes que avaliam, segundo a perspectiva do paciente, o impacto do DM na qualidade de vida e nos aspectos emocionais (depressão, culpa, raiva e medo). Cada item do questionário utiliza a escala de Likert de cinco pontos, variando de um a seis, em que um significa "nenhum problema" e seis, "problema sério", e produz escore total de 0 a 100 , medido pela soma das respostas (0-4) multiplicada por 1,25. O escore 
mais alto indica alto nível de sofrimento emocional (GROSS et al., 2007; AGUIAR et al., 2008; CURCIO; LIMA; ALEXANDRE, 2011).

O DQOL (Diabetes Quality of Life Measure) apresenta confiabilidade adequada para sua aplicação e mostra-se um dos questionários mais utilizados para avaliar a qualidade de vida relacionada à saúde de diabéticos. É composto de 46 itens de múltipla escolha com quatro domínios (satisfaçáo, impacto, preocupação social/vocacional e preocupação relacionada com o DM). Cada questão utiliza uma escala de um a cinco pontos. Classifica-se como o melhor resultado quando a resposta estiver mais próxima de um. Dentro do domínio de satisfaçáo, a resposta um significa "muito satisfeito" e cinco, "nada satisfeito"; já nos domínios de preocupação e impacto, um sugere "nunca" e cinco, "sempre" (CORRER et al., 2008).

O QAD (Questionário de Atividades de Autocuidado com o Diabetes) abrange seis dimensóes com 15 itens: alimentação geral, alimentação específica, atividade física, monitorização glicêmica, cuidados com os pés e medicação, e três itens relacionados ao tabagismo, totalizando 18 perguntas. Os dados são analisados de acordo com a aderência aos itens, parametrizados em dias de semana. Durante a avaliação, devem responder à frequência com que realizam as atividades ou comportamentos nos sete últimos dias. Tais respostas devem variar entre zero e sete, em que zero significa "situação menos desejável" e sete, a "mais favorável". Porém, na dimensão "alimentação específica", os valores são invertidos. Os itens relacionados ao tabagismo levam em conta a proporção de fumantes, a média de consumo do cigarro e, ainda, a última vez em que o individuo fumou (MICHELS et al., 2010).

Para a análise estatística, foi aplicado o teste de Shapiro-Wilk para analisar a normalidade dos valores. Os dados com distribuição normal foram apresentados em média e desvio-padrão e os não normais, em mediana e percentis. Os dados nominais foram apresentados em frequências absoluta e relativa (\%). Para correlacionar o tempo de diagnóstico de DM com escores dos questionários B-PAID e DQOL, aplicou-se a correlação de Spearman. O nível de significância adotado foi de p inferior a 0,05.

\section{Resultados}

Participaram do estudo 60 pacientes com diagnóstico de DM2 e foi excluído um sujeito devido ao tempo de diagnóstico de DM ser inferior a um ano (cinco meses). A amostra final foi constituída de 59 indivíduos, sendo 20 (33,9\%) homens e $39(66,1 \%)$ mulheres, com idade de 63,5 \pm 10 anos, $46(78,7 \%)$ eram da etnia branca, 37 (62,7\%), casados, 42 (71,2\%) viviam com companheiro(a) e 20 (34\%) apresentavam Ensino Fundamental incompleto.

Dezoito pacientes $(30,5 \%)$ apresentaram diagnóstico de DM2 de um a cinco anos, 14 (23,7\%), de seis a dez anos, e 27 (45,8\%), havia mais de dez anos.

A média do peso dos pacientes foi $79,3 \pm 13 \mathrm{~kg}$, altura de $1,60 \pm 0,1 \mathrm{~m}$ e IMC de 29,3 $\pm 4,4 \mathrm{~kg} / \mathrm{m}^{2}$. Em relação à medida da circunferência abdominal (CA), a média das mulheres foi de 101,4 $\pm 12 \mathrm{~cm}$ e a dos homens, de $104 \pm 10 \mathrm{~cm}$. Todas as mulheres e $80 \%$ dos homens estavam com valores acima do normal, indicando risco cardiovascular aumentado de acordo com as Diretrizes Brasileiras de Obesidade (ASSOCIAÇÃO..., 2010). O tempo de tabagismo relatado pelos pacientes foi de um a 52 anos. Trinta e sete indivíduos (63\%) apresentaram histórico familiar de DM. Quanto às doenças associadas, $58 \%$ dos indivíduos apresentaram hipertensão arterial sistêmica (HAS) e 10\%, HAS e dislipidemia. Outros fatores de risco para doença cardiovascular encontram-se na Tabela 1.

Em relação às respostas dos questionários, foram apresentadas aquelas que indicaram maior impacto causado pelo DM na vida do indivíduo de modo

Tabela 1. Fatores de risco para doença cardiovascular.

\begin{tabular}{cccc}
\hline Variáveis & & $\mathbf{n}$ & $\mathbf{( \% )}$ \\
\hline \multirow{2}{*}{ Antecedentes familiares } & Sim & 37 & 62,7 \\
\cline { 2 - 4 } & Não & 22 & 37,3 \\
\hline \multirow{2}{*}{ Tabagismo } & Sim & 4 & 6,8 \\
\cline { 2 - 4 } & Não & 40 & 67,8 \\
\cline { 2 - 4 } & Ex & 15 & 25,4 \\
\hline \multirow{2}{*}{ Sedentarismo } & Sim & 31 & 52,5 \\
\cline { 2 - 4 } & Não & 28 & 47,5 \\
\hline \multirow{2}{*}{ Peso } & Ideal & 10 & 17 \\
& Sobrepeso & 24 & 40,6 \\
\cline { 2 - 4 } & Obesidade & 25 & 42,4 \\
\hline
\end{tabular}

Dados expressos em frequências absoluta (n) e relativa (\%). 
geral. No questionário B-PAID, o escore mediano foi de 19 (11 a 48) pontos. Apenas dois pacientes tiveram o escore mínimo (zero), indicando não terem sofrimento emocional com a doença, e somente um paciente apresentou escore máximo (100), indicando alto nível de sofrimento emocional. $\mathrm{Na}$ Tabela 2 estão as questôes que mostraram a maior porcentagem de indivíduos que consideraram o DM "um problema sério" na vida.

Quanto ao questionário DQOL, na Tabela 3 estão os valores em porcentagem dos indivíduos que relataram pior resultado referente à satisfação, maior impacto e maior intensidade de preocupação devido ao DM, indicando, assim, elementos que permitem inferir a influência causada pela doença na qualidade de vida.

No QAD, foi possível encontrar menor aderência para o item "Ingerir doces" ( $2 \pm 2$ dias por semana) e maior para o item "Tomar o número indicado de comprimidos do diabetes" ( $7 \pm 1$ dias por semana). Todos os dados obtidos estáo expressos na Tabela 4.

Houve correlação positiva do escore do questionário B-PAID com o tempo de diagnóstico de DM ( $\mathrm{p}<0,001)$, mostrando que quanto menor o tempo, menor o sofrimento emocional relacionado à doença (Figura 1). Não foi encontrada diferença estatística na correlaçáo do tempo com o escore do questionário DQOL $(\mathrm{p}=0,08)$.

\section{Discussão}

No presente trabalho foram aplicados três questionários específicos para pacientes diabéticos, visando avaliar aspectos físicos, emocionais, sociais e qualidade de vida.

O maior número de indivíduos foi do sexo feminino. Conforme os dados do Instituto Brasileiro de Geografia e Estatítica (2012), 44,3\% da população idosa é masculina, enquanto $55,7 \%$ é feminina. Há uma concentraçáo de mulheres nesse grupo etário. A razáo de sexos para a população com mais de 60 anos de idade é de cerca de 0,8 , indicando que existem aproximadamente 80 homens para cada 100 mulheres, resultado dos diferenciais de mortalidade entre os sexos, cujas taxas para a população masculina são sempre maiores do que aquelas observadas entre as mulheres. Dessa forma, as mulheres vivem, em média, oito anos mais que os homens, levando a uma predominância do sexo feminino na população idosa, fenômeno conhecido como "feminização da velhice" (CARAMANO, 2006; NERI, 2008; VIRTUOSO JÚNIOR; GUERRA, 2008).

Tabela 2. Questões de Problems Areas in Diabetes (B-PAID) com maior porcentagem de indivíduos que consideraram o DM "um problema sério".

\begin{tabular}{lc}
\hline \multicolumn{1}{c}{ Questões } & Indivíduos (\%) \\
\hline A falta de metas claras e concretas no cuidado do seu diabetes & 20 \\
Sentir que seu diabetes é um peso para você & 20 \\
Preocupar-se com episódios de glicose baixa & 27 \\
Preocupar-se com a comida e o que comer & 20 \\
Preocupar-se com o futuro e a possibilidade de sérias complicações & 42 \\
Não aceitar seu diabetes & 19
\end{tabular}

Dados expressos em frequência relativa (\%).

Tabela 3. Questões do Diabetes Quality of Life Measure (DQOL) com os piores resultados referentes à satisfação, maior impacto e maior intensidade de preocupação devido a DM.

\begin{tabular}{lc}
\hline \multicolumn{1}{c}{ Domínio de satisfação } & Nada satisfeito (\%) \\
\hline Você está satisfeito(a) com seu sono? & 20 \\
Você está satisfeito(a) com a aparência do seu corpo? & 20 \\
Você está satisfeito(a) com o que gasta fazendo exercícios físicos? & 31 \\
Você está satisfeito(a) com seu tempo de lazer? & 24 \\
\hline \multicolumn{1}{c}{ Domínio impacto } & Maior impacto (\%) \\
\hline Com que frequência você se sente incomodado(a) por ter diabetes? & 19 \\
Com que frequência você sente que, por causa do diabetes, vai ao banheiro mais que & 24 \\
os outros? $\quad$ Domínio preocupações relacionadas ao diabetes mellitus & Sempre (\%) \\
\hline Com que frequência você se preocupa se virá a desmaiar? & 17 \\
Com que frequência se preocupa se terá complicações devidas a seu diabetes? & 36 \\
\hline
\end{tabular}

Dados expressos em frequência relativa (\%). 
Tabela 4. Questionário de Atividades de Autocuidado com o Diabetes (QAD).

\begin{tabular}{lc}
\hline \multicolumn{1}{c}{ Itens } & Aderência (em dias) \\
\hline Seguiu uma dieta saudável & $5 \pm 2$ \\
Seguiu uma orientação alimentar & $4 \pm 3$ \\
Ingerir cinco ou mais porções de frutas e/ou vegetais & $5 \pm 3$ \\
Ingerir carne vermelha e/ou derivados de leite integral & $3 \pm 2$ \\
Ingerir doces & $2 \pm 2$ \\
Realizar atividades físicas por pelo menos 30 minutos & $2 \pm 3$ \\
Realizar atividades físicas específicas (caminhar, nadar etc.) & $2 \pm 3$ \\
Avaliar o açúcar no sangue & $3 \pm 3$ \\
Avaliar o açúcar no sangue o número de vezes recomendado & $3 \pm 3$ \\
Examinar os pés & $4 \pm 3$ \\
Examinar dentro dos sapatos antes de calçá-los & $3 \pm 3$ \\
Secar os espaços entre os dedos dos pés depois de lavá-los & $6 \pm 2$ \\
Tomar injeções de insulina conforme recomendado & $2 \pm 3$ \\
Tomar o número indicado de comprimidos do diabetes & $7 \pm 1$ \\
\hline
\end{tabular}

Dados expressos em média \pm desvio-padrão.

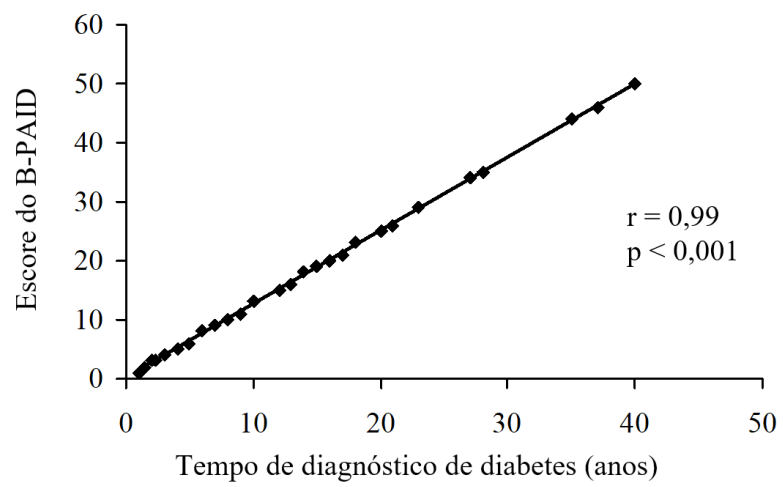

Figura 1. Correlação do escore do questionário B-PAID com tempo de diagnóstico de DM.

No estudo de Torres et al. (2010), foram avaliados 105 indivíduos com DM, com idade entre 30 e 79 anos e, destes, 75,2\% eram do sexo feminino com maior frequência de pessoas com mais de 50 anos de idade. Zaitune et al. (2006) estudaram 426 sujeitos com mais de 60 anos e constataram que as mulheres têm maior probabilidade de desenvolver HAS e/ou DM por apresentarem melhor percepção das doenças devido ao autocuidado e por buscarem mais assistência médica do que os homens. Miranzi et al. (2008) concordaram e chamaram atenção, afirmando que a maioria de mulheres nos estudos pode estar relacionada ao fato de existir uma maior população feminina no planeta. Duarte e Uchôa-Figueiredo (2010) corroboraram esses achados, observando prevalência do sexo feminino.

No presente estudo, a frequência de sobrepeso e obesidade foi elevada ( $83 \%$ dos indivíduos). Tal fator de risco é modificável e encontra-se consideravelmente aumentado na população, considerando IMC igual ou superior a $25 \mathrm{~kg} / \mathrm{m}^{2}$ (ASSOCIAÇÃO..., 2010). Silva et al. (2006), em seu estudo com uma amostra de 89 indivíduos, funcionários da Embrapa com sede em Londrina (PR), idade entre 20 e 65 anos, constataram que a obesidade está associada à HAS e ao DM, tendo em vista que o excesso de gordura participa efetivamente do desequilíbrio metabólico, aumentando, assim, os índices de morbimortalidade. Considerando que as doenças cardiovasculares podem ter o mesmo componente genético e ambiental que o DM, comprova-se a hipótese da associação de HAS com DM (MCLELLAN et al., 2007).

A combinação do aumento de IMC e CA torna-se mais eficiente na predição de patologias cardiovasculares do que isoladamente. Cavalcanti et al. (2009), em uma revisão sistemática sobre obesidade, com publicaçôes no período de 1993 a 2007, associaram esses fatores às variáveis alimentares e à atividade física, o que pode ser comprovado neste estudo, em que $52,5 \%$ dos indivíduos eram sedentários. O sedentarismo 
pode ser um fator de risco tão importante quanto a alimentaçáo inadequada, podendo aumentar o índice de DM2 (MCLELLAN et al., 2007).

A literatura afirma que DM é frequentemente acompanhado de dislipidemia, HAS e disfunção endotelial (MCLELLAN et al., 2007). Lima et al. (2011) estudaram idosos diabéticos adscritos em um Programa de Saúde da Família do município de Belo Horizonte, com 60 a 91 anos, sendo a maioria do sexo feminino, e encontraram HAS como a principal comorbidade relacionada a DM. Esses achados se confirmam neste estudo, pois HAS estava presente em 57,6\% dos diabéticos estudados.

A pesquisa Vigilância de Fatores de Risco e Proteção para Doenças Crônicas por Inquérito Telefônico (BRASIL, 2013) comprovou que o avanço do DM está relacionado a excesso de peso (75\% dos indivíduos com DM estavam acima do peso), falta de exercícios físicos, má alimentação e envelhecimento da população.

O presente estudo corrobora dados apresentados por Gross et al. (2004), Souza et al. (2012) e Freitas et al. (2014), com predominância do sexo feminino, média de idade de 60 anos e baixa escolaridade. A amostra do estudo de Lima et al. (2011) teve predominância de $48,7 \%$ dos indivíduos com Ensino Fundamental incompleto, resultado similar a este estudo. Esse fato pode dificultar a compreensão da doença e os cuidados com a saúde.

A maior prevalência de DM no presente estudo foi em indivíduos que vivem com companheiro(a), sem filhos, corroborando o estudo transversal de Belon et al. (2008), com amostra de 1.949 idosos não institucionalizados, maioria do sexo feminino, residentes nos municípios de Botucatu, Itapecerica da Serra, Embu, Campinas, Taboão da Serra e Butantá, estado de São Paulo, sugerindo que a companhia do cônjuge favorece o acesso ao serviço de saúde e o autocuidado.

O ato de "cuidar da saúde" remete ao conceito canadense de empowerment, embora de difícil tradução para a língua portuguesa, dá a ideia de garantir ao sujeito o controle de sua vida, tornando-o capaz de lidar com as limitaçóes impostas pelo DM, fundamental para o sucesso do tratamento e a qualidade de vida (TAVARES, 2006).

O escore mediano analisado com B-PAID foi de 19 pontos, significando baixo sofrimento emocional e concordando com as pesquisas de Miranda et al. (2010) e Souza et al. (2012), que utilizaram o mesmo questionário com 17 pacientes cadastrados em ESF de Minas Gerais e 170 pacientes cadastrados no Ambulatório de Endocrinologia da Universidade
Federal de Sergipe, com escores médio de 17,2 e abaixo de 40 , respectivamente, revelando baixo sofrimento.

O tratamento do DM envolve tanto mudanças no estilo de vida quanto introdução de numerosos medicamentos que podem aumentar com a presença de complicações (FERREIRA et al., 2013). No presente estudo, verificou-se que grande parte dos pacientes apresentou tempo de diagnóstico entre 10 e 20 anos (44\%), dados semelhantes ao estudo de Tavares et al. (2007), constituído por 113 indivíduos do município de Uberaba (MG), em que 51,3\% apresentavam diagnóstico entre 10 e 20 anos. Ainda referente ao presente estudo, no questionário B-PAID, $42 \%$ relataram "preocupar-se com o futuro e a possibilidade de sérias complicaçôes", o que confirma a correlação positiva observada entre o tempo de diagnóstico de DM e o escore do B-PAID, que indica sofrimento. Ferreira et al. (2013) afirmam que quanto maior o tempo de diagnóstico, quando as complicaçôes agravam e intensificam o tratamento, os indivíduos relatam mais angústia e sentem-se mais vulneráveis.

No questionário DQOL, uma das preocupações mais frequentes relatadas pelos participantes do estudo foram complicaçôes do DM, podendo significar que há conhecimento dessas complicaçóes, o que pode colaborar para um tratamento adequado da doença. Tal dado também foi apresentado no artigo de Jacom et al. (2012), que aplicou o questionário em 87 pacientes com DM2 da UBS Nilton Luiz de Castro, em Ponta Grossa (PR).

O QAD avalia a aderência do paciente ao autocuidado e ele responde em quantos dos últimos sete dias realizou determinada atividade ou comportamento (MICHELS et al., 2010). Os resultados observados para essa variável discordam do estudo de Freitas et al. (2014), que avaliou 96 indivíduos cadastrados na estratégia de Saúde da Família (ESF) do município de Teresina (PI), em que a maior aderência foi comer cinco ou mais porçóes de frutas ou vegetais e a menor aderência foi seguir as orientações nutricionais. Também discordam de Santos (2013), que estudou pacientes cadastrados no programa Hiperdia, nas UBS de Itajubá (MG), e mostrou baixa aderência relacionada ao autocuidado com alimentação e alto consumo de açúcar e gorduras saturadas. No presente estudo, a maior aderência foi tomar o número indicado de comprimidos e a menor aderência foi ingerir doces e gorduras. Observou-se que independentemente do tempo de diagnóstico do DM, o cuidado maior dos pacientes foi em relação ao uso correto dos medicamentos e o segundo maior foi o cuidado com os pés. 


\section{Conclusão}

Ao aplicar os questionários, no B-PAID se encontrou baixo sofrimento emocional relacionado ao DM, no DQOL, preocupação frequente com complicaçôes causadas pela doença, e no QAD, boa aderência em relaçáo ao uso dos medicamentos e baixa aderência em relação à ingestão de doces e gorduras. Além disso, houve correlação positiva significante do tempo de diagnóstico de DM com o escore do B-PAID. As evidências explicitadas com a investigaçáo de um grupo de indivíduos apontam para a superação de um modelo de intervenção assimétrico, que desconsidera o complexo universo psicológico das pessoas acometidas por doenças crônicas. Neste estudo, assim como em muitos outros aqui referendados, um modelo interventivo mais relacional e sensível aos aspectos emocionais aponta para mudanças necessárias, especialmente na concepçáo de que as pessoas devem ser consideradas ativas no processo de conhecer suas características, limites e possibilidades ante o quadro em que se encontram.

Espera-se que este estudo contribua para direcionar intervenções de saúde e trazer informaçóes sobre os pacientes, visando melhorar a qualidade de vida e o autocuidado do DM2.

\section{Referências}

AGUIAR, C. T. et al. Instrumentos de avaliaçấo de qualidade de vida relacionada à saúde no diabetes mellitus. Arquivos Brasileiros de Endocrinologia e Metabologia, São Paulo, v. 52, n. 6, p. 52-56, 2008.

ARSA, G. et al. Diabetes Mellitus tipo 2: Aspectos fisiológicos, genéticos e formas de exercício físico para seu controle. Revista Brasileira de Cineantropometria \& Desempenho Humano, Florianópolis, v. 11, n. 1, p. 103111, 2009.

ASSOCIAÇÃOO BRASILEIRA PARA O ESTUDO DA OBESIDADE E DA SÍNDROME METABÓLICA ABESO. Diretrizes Brasileiras de Obesidade 2009-2010. Itapevi: AC Farmacêutica, 2010.

ASSOCIAÇÃO NACIONAL DE ASSISTÊNCIA AO DIABÉTICO - ANAD. O que deve saber sobre o diabetes. São Paulo, 2015. Disponível em: <http://www.anad. org.br/>. Acesso em: 21 set. 2015.

BELON, A. P. et al. Diabetes em idosos: perfil sócio-demográfico e uso de serviços de saúde. In: ENCONTRO NACIONAL DE ESTUDOS POPULACIONAIS, 16., 2008, Caxambu. Anais... São Paulo: ABEP, 2008. p. $1-10$.

BRASIL. Ministério da Saúde. Secretaria de Vigilância em Saúde. Departamento de Vigilância de Doenças e Agravos não Transmissíveis e Promoçáo de Saúde. Vigitel Brasil 2012: vigilância de fatores de risco e proteçáo para doenças crônicas por inquérito telefônico. Brasília: Ministério da Saúde, 2013.

BRASIL. Ministério da Saúde. Departamento de Informática do SUS - DATASUS. Prevalência de diabete melito. Brasília: DATASUS, 2015. Disponível em: <http:// tabnet.datasus.gov.br/cgi/tabnet.exe?idb2012/g01.def> . Acesso em: 13 fev. 2015.

CARAMANO, A. A. Envelhecimento da população brasileira: uma contribuição demográfica. Tratado de geriatria e gerontologia. Rio de Janeiro: Guanabara Koogan, 2006.

CAVALCANTI, C. B. S.; CARVALHO, S. C. B. E.; BARROS, M. V. G. Indicadores antropométricos de obesidade abdominal: revisão dos artigos indexados na biblioteca SciELO. Revista Brasileira de Cineantropometria \& Desempenho Humano, Florianópolis, v. 11, n. 2, p. 217-225, 2009.

CORDEIRO, J. J. R. Cardiologia. In: CAVALCANTI, A.; GALVÃO, C. Terapia Ocupacional: fundamentaçāo \& prática. Rio de Janeiro: Guanabara Koogan, 2007. p. 502-507.

CORRER, C. J. et al. Tradução para o Português e Validaçáo do Instrumento Diabetes Quality of Life Measure (DQOL-Brasil). Arquivos Brasileiros de Endocrinologia e Metabologia, São Paulo, v. 52, n. 3, p. 515-522, 2008.

CORRER, R. et al. Avaliaçáo do cotidiano e enfrentamento de adolescentes com diabetes mellitus 1. Salusvita, Bauru, v. 32, n. 3, p. 243-263, 2013.

CURCIO, R.; LIMA, M. H. M.; ALEXANDRE, N. M. C. Instrumentos relacionados ao diabetes mellitus adaptados e validados para a cultura brasileira. Revista Eletrônica de Enfermagem, Goiânia, v. 13, n. 2, p. 331337, 2011.

DUARTE, G. P.; UCHÔA-FIGUEIREDO, L. R. A vida cotidiana e a qualidade de vida de pacientes atendidos na atenção primária de saúde. Cadernos de Terapia Ocupacional da UFSCar, São Carlos, v. 18, n. 1, p. 1933, 2010.

DUNCAN, B. B. et al. Doenças crônicas não transmissíveis no Brasil: prioridade para enfrentamento e investigação. Revista de Saúde Pública, São Paulo, v. 46, p. 126-134, 2012. Suplemento.

FERREIRA, D. S. P. et al. Repercussão emocional diante do diagnóstico de diabetes mellitus tipo 2. Revista Enfermagem UERJ, Rio de Janeiro, v. 21, n. 1, p. 41-46, 2013.

FREITAS, S. S. et al. Analysis of the self-care of diabetics according to by the Summary of Diabetes Self-Care Activities Questionnaire (SDSCA). Acta Scientiarum. Health Sciences, Maringá, v. 36, n. 1, p. 73-81, 2014.

GROSS, C. C. et al. Brazilian version of the problem areas in diabetes scale (B-PAID): validation and identification of individuals at high risk for emotional distress. Diabetes Research and Clinical Practice, Oxford, v. 76, n. 3, p. 455-459, 2007. 
GROSS, C. C. et al. Versäo brasileira da escala PAID (Problem areas in diabetes): avaliaçẫo do impacto do diabetes na qualidade de vida. 2004. 60 f. Dissertação (Mestrado em Psicologia do desenvolvimento) - Universidade Federal do Rio Grande do Sul, Porto Alegre, 2004.

INSTITUTO BRASILEIRO DE GEOGRAFIA E ESTATÍSTICA - IBGE. Mulheres são maioria na população e ocupam mais espaço no mercado de trabalho. Rio de Janeiro, 2012. Disponível em: <http://www.brasil.gov. br/cidadania-e-justica/2015/03/mulheres-sao-maioria-da-populacao-e-ocupam-mais-espaco-no-mercado-de-trabalho>. Acesso em: 18 fev. 2015.

INTERNATIONAL DIABETES FEDERATION IDF. Key findings. Brussels: IDF, 2014. Disponível em: $<$ http://www.idf.org/diabetesatlas/update-2014>. Acesso em: 20 fev. 2015.

JACOM, T. et al. Aplicação do questionário de qualidade de vida DQOL-Brasil a pacientes portadores de Diabetes Mellitus tipo 2 na Unidade Básica de Saúde Nilton Luiz de Castro - Ponta Grossa-PR. In: ENCONTRO CONVERSANDO SOBRE EXTENSÃO NA UEPG - CONEX, 11., 2012, Ponta Grossa. Anais... Ponta Grossa: UEPG, 2012. p. 1-6.

LIMA, A. P.; PEREIRA, D. A. G.; ROMANO, V. F. Perfil sócio-demográfico e de saúde de idosos diabéticos atendidos na Atenção Primária. Revista Brasileira de Ciências da Saúde, São Caetano do Sul, v. 15, n. 1, p. 3946, 2011.

MCLELLAN, K. C. P. et al. Diabetes mellitus do tipo 2, síndrome metabólica e modificação no estilo de vida. Revista de Nutrição, Campinas, v. 20, n. 5, p. 515-524, 2007.

MICHELS, M. J. et al. Questionário de Atividades de Autocuidado com o Diabetes: tradução, adaptação e avaliação das propriedades psicométricas. Arquivos Brasileiros de Endocrinologia e Metabologia, São Paulo, v. 54, n. 7, p. 644-651, 2010.

MIRANDA, L. P. et al. Qualidade de vida de idosos com Diabetes Mellitus cadastrados na estratégia saúde da família. Revista Mineira de Educação Física, Viçosa, n. 5, p. 125-135, 2010.

MIRANZI, S. S. C. et al. Qualidade de vida de indivíduos com diabetes mellitus e hipertensão acompanhados por uma equipe de saúde da família. Texto \& Contexto - Enfermagem, Florianópolis, v. 17, n. 4, p. 672-629, 2008.

NERI, A. L. Paradigmas contemporâneos sobre o desenvolvimento humano em psicologia e em sociologia. In: NERI, A. L. Desenvolvimento e envelhecimento: perspectivas biológicas, psicológicas e sociológicas. Campinas: Papirus, 2008. p. 11-37.

ORGANIZAÇÃO MUNDIAL DA SAÚDE - OMS. Doenças transmissiveis e não transmissiveis. Brasilia, 2010. Disponível em: <http://www.paho.org/bra/index. php?option=com_content\&view=article\&id=394:diabe tes-mellitus\&Itemid=539>. Acesso em: 13 fev. 2015.
SANTOS, A. M. Relação entre o tempo do diagnóstico de Diabetes Mellitus tipo II e o autocuidado dos pacientes de um município de Minas Gerais. Itajubá: Escola de Enfermagem Wenceslau Braz, 2013. Relatório de Iniciaçáo Científica.

SILVA, G. B. M.; SKARE, L. T. Manifestaçóes musculoesqueléticas em diabetes mellitus. Revista Brasileira de Reumatologia, São Paulo, v. 52, n. 4, p. 594-609, 2012.

SILVA, J. L. T. et al. Distribuição centrípeta da gordura corporal, sobrepeso e aptidão cardiorrespiratória: associação com sensibilidade insulínica e alteraçôes metabólicas. Arquivos Brasileiros de Endocrinologia e Metabologia, São Paulo, v. 50, n. 6, p. 1034-1040, 2006.

SOCIEDADE BRASILEIRA DE DIABETES - SBD. Diabetes Tipo 2. São Paulo, 2014a. Disponível em: $<$ http://www.diabetes.org.br/diabetes-tipo-2>. Acesso em: 16 fev. 2015.

SOCIEDADE BRASILEIRA DE DIABETES - SBD. $O$ avanço do diabetes no mundo, segundo a OMS. São Paulo, 2014b. Disponível em: <http://www.diabetes.org.br/ ultimas/o-avanco-do-diabetes-no-mundo-segundo-a-oms>. Acesso em: 13 fev. 2015.

SOUZA, E. C. S. et al. Avaliação da qualidade de vida de portadores de diabetes utilizando a medida específica B-PAID. Revista Mineira de Enfermagem, Viçosa, v. 16, n. 4, p. 509-514, 2012.

TANAKA, O. Y.; MELO, C. Uma proposta de abordagem transdisciplinar para avaliação em saúde. Interface - Comunicação, Saúde, Educação, Botucatu, v. 4, n. 7, p. 113-118, 2000

TAVARES, D. M. S. et al. Caracterização de idosos diabéticos atendidos na atençáo secundária. Ciência \& Saúde Coletiva, Rio de Janeiro, v. 12, n. 5, p. 1341-1352, 2007.

TAVARES, G. S. A influência do estilo de vida na re-internação hospitalar de pacientes diabéticos. 2006. $141 \mathrm{f}$ Dissertação (Mestrado em Ciências Médicas) - Universidade de São Paulo, Ribeirão Preto, 2006.

TORRES, H. C.; PACE, A. E.; STRADIOTO, M. A. Análise sociodemográfica e clínica de indivíduos com Diabetes tipo 2 e sua relação com o autocuidado. Cogitare Enfermagem, Curitiba, v. 15, n. 1, p. 48-54, 2010.

VIRTUOSO JÚNIOR, J. S.; GUERRA, R. O. Fatores associados às limitaçóes funcionais em idosas de baixa renda. Revista da Associação Médica Brasileira, São Paulo, v. 54, n. 5, p. 430-435, 2008. http://dx.doi. org/10.1590/S0104-42302008000500017.

ZABAGLIA, R. et al. Efeito dos exercícios resistidos em portadores de diabetes mellitus. Revista Brasileira de Prescrição e Fisiologia do Exercício, Maranhão, v. 3, n. 18, p. 547-558, 2009

ZAITUNE, M. P. A. et al. Hipertensão Arterial em idosos: prevalência, fatores associados e práticas de controle no município de Campinas, São Paulo, Brasil. Cadernos de Saúde Pública, Rio de Janeiro, v. 22, n. 2, p. 285-294, 2006. 


\section{Contribuição dos Autores}

Luciana Sabadini Bernini: participou da concepção e planejamento do trabalho, da interpretação dos resultados, redação e revisão das versóes preliminares e definitiva. Silvia Regina Barrile, Eduardo Aguilar Arca, Rinaldo Correr, Maria Amélia Ximenes, Débora Neves: participou da análise e interpretação dos resultados e redação das versôes preliminares e definitiva. Aline: participou da coleta de dados, interpretação dos resultados e redação. Camila Gimenes: autora principal, participou da concepção e planejamento do trabalho, bem como da interpretaçáo dos resultados, redação e revisão das versóes preliminares e definitiva. Todos os autores aprovaram a versão final do texto. 